\title{
ON THE RELATIONSHIP BETWEEN DENSITY AND WEAK DENSITY IN BOOLEAN ALGEBRAS
}

\author{
KYLE BOZEMAN
}

(Communicated by Andreas R. Blass)

\begin{abstract}
Given a homogeneous, complete Boolean algebra $B$, it is shown that $\mathrm{d}(B) \leq \min \left(2^{<\mathrm{wd}(B)}, \sup \left\{\lambda^{c(B)}: \lambda<\operatorname{wd}(B)\right\}\right)$ in $\mathrm{ZFC}$, where $\mathrm{d}(B)$ is the density, $\operatorname{wd}(B)$ is the weak density, and $c(B)$ is the cellularity of $B$. A corollary to this result is that $\mathrm{d}(B)=\mathrm{wd}(B)$ in $\mathrm{ZFC}+\mathrm{GCH}$.
\end{abstract}

\section{INTRODUCTION AND PRELIMINARIES}

Throughout this paper, $B$, or more properly $(B,+, \cdot,-, 0,1)$, denotes a homogeneous, complete Boolean algebra and $|X|$ denotes the cardinality of a set $X$. A subset $X$ of $B$ is dense if and only if $\forall a \in B \backslash\{0\}, \exists$ nonzero $x \in X$ such that $x \leq a$. A subset $X$ of $B$ is weakly dense if and only if $\forall a \in B \backslash\{0\}, \exists$ nonzero $x \in X$ such that $x \leq a$ or $x \leq-a$. The density of $B$ is defined by $\mathrm{d}(B)=\min \{|X|: X$ is dense $\}$. The weak density of $B$ is defined by $\operatorname{wd}(B)=\min \{|X|: X$ is weakly dense $\}$. Clearly, $\operatorname{wd}(B) \leq \mathrm{d}(B)$. Recently, there has been interest in determining a bound for $\mathrm{d}(B)$ in terms of $\operatorname{wd}(B)$. M. Burke has shown $\operatorname{wd}(B)=\mathrm{d}(B)$ for measure algebras [2]. Recall that $X$ is an antichain means $X \subset B \backslash\{0\}$ and consists of pairwise disjoint elements and the cellularity of $B, c(B)=\sup \{|A|: A$ is an antichain $\}$ [4]. W. Just has shown that $\operatorname{wd}(B)=\mathrm{d}(B)$ in $\mathrm{ZFC}+\mathrm{GCH}$ for c.c.c. algebras (i.e., algebras for which $c(B)$ is countable) [3]. We will establish that

$$
\mathrm{d}(B) \leq \min \left(2^{<\operatorname{wd}(B)}, \sup \left\{\lambda^{c(B)}: \lambda<\operatorname{wd}(B)\right\}\right)
$$

in ZFC. It immediately follows from this inequality that $\operatorname{wd}(B)=\mathrm{d}(B)$ in $\mathrm{ZFC}+\mathrm{GCH}$.

Concerning notation and terminology, $X$ will always indicate a subset of $B$, $X^{+}=X \backslash\{0\}, X \mid a=\{x \cdot a \mid x \in X\}$, and $a$ and $b$ are disjoint means $a \neq b$ and $a \cdot b=0 . X$ is said to be homogeneously weakly dense if and only if for each $a$ in $B^{+}, X \mid a$ is weakly dense in $B \mid a$. The homogeneous weak density

Received by the editors April 2, 1990 and, in revised form, April 23, 1990.

1980 Mathematics Subject Classification (1985 Revision). Primary 03E35.

Key words and phrases. Dense, weakly dense, homogeneously weakly dense, nowhere relatively dense, cellularity. 
of $B, \operatorname{hwd}(B)=\min \{|X|: X$ is homogeneously weakly dense $\} . X$ is nowhere relatively dense if and only if for each $a$ in $B^{+}, X \mid a$ is not dense in $B \mid a$. The concentration of $X$ at $a$ is defined by $\operatorname{con}(X, a)=\{x \in X: x \cdot a \neq 0\}$. The subalgebra generated by $X$ is denoted by $\mathscr{A}(X)$. For a function $f: G \rightarrow H$, $f^{\prime \prime}(G)=\{f(g): g \in G\}$. Throughout, we use standard set-theoretic notation, e.g., $\omega$ denotes the set of nonnegative integers.

Observation 1.1. $X$ is weakly dense if and only if for all $a$ in $B \operatorname{con}(X, a) \neq$ $\operatorname{con}(X,-a)$.

Observation 1.2. $X$ is homogeneously weakly dense if and only if whenever $a$ and $b$ are disjoint, $\operatorname{con}(X, a) \neq \operatorname{con}(X, b)$.

Proof. If $X$ is homogeneously weakly dense and $a$ and $b$ are disjoint, then $X \mid(a+b)$ is weakly dense in $B \mid(a+b)$ in which case $\operatorname{con}(X, a) \neq \operatorname{con}(X, b)$, by Observation 1.1. Conversely, suppose $\forall$ disjoint $a, b \in B, \operatorname{con}(X, a) \neq$ $\operatorname{con}(X, b)$. Let $c \in B^{+}$. Then $\forall d \in B, \operatorname{con}(X, c \cdot d) \neq \operatorname{con}(X, c \cdot(-d))$. By Observation 1.1, $X \mid c$ is weakly dense in $B \mid c$. Thus, $X$ is homogeneously weakly dense.

Observation 1.3. $X$ is nowhere relatively dense if and only if for each a in $B^{+}$, there is some nonzero $d<a$ such that $\operatorname{con}(X, d) \subset \operatorname{con}(X, a-d)$.

\section{HOMOGENEOUS WEAK DENSITY}

If a subset is nowhere relatively dense, is it "sparse" enough to exclude the condition of being weakly dense? In other words, if a subset $X$ is weakly dense does there necessarily exist $a$ in $B^{+}$such that $X \mid a$ is dense in $B \mid a$ ? The answer is "no." If one considers collapsing algebras there may be constructed a weakly dense set which is at the same time nowhere relatively dense [1].

In this section, we answer a similar question concerning the notion of homogeneously weakly dense. The condition of being homogeneously weakly dense is strictly stronger than that of being weakly dense. For example, let $a \in B \backslash\{1,0\}$. Note that $B \mid a$ is weakly dense in $B$ but not homogeneously weakly dense in $B$. However, the following theorem does hold.

Theorem 2.1. Let $X$ be weakly dense. There exists $c \in B^{+}$such that $X \mid c$ is homogeneously weakly dense in $B \mid c$. Consequently, $\operatorname{hwd}(B)=\operatorname{wd}(B)$.

Proof. We assume $X \mid c$ is not homogeneously weakly dense in $B \mid c$ for all $c \in$ $B^{+}$. Then there is a dense set of $a \in B^{+}$such that $X \mid a$ is not weakly dense in $B \mid a$. Hence there is a maximal antichain of such $a$. Now for each $a \in A$ write $a=a_{0}+a_{1}$ where $a_{0} \cdot a_{1}=0$ and $\operatorname{con}\left(X, a_{0}\right)=\operatorname{con}\left(X, a_{1}\right)$. Then $\sum_{a \in A} a_{0}$ and $\sum_{a \in A} a_{1}$ both meet every member of $X$, contradicting the weak density of $X$. The fact that $\operatorname{hwd}(B)=\operatorname{wd}(B)$ follows from the homogeneity of $B$.

\section{MAIN RESULT}

Throughout this section, let $X=\left(x_{\tau}\right)_{\tau<\kappa}$ be a homogeneously weakly dense set with $|X|=\operatorname{wd}(B)=\kappa$. For each $\rho<\kappa, Y_{\rho}$ denotes $\left(x_{\tau}\right)_{\tau \leq \rho} . W$ is a 
bounded subset of $X$ means $\exists \rho<\kappa$ such that $W \subset Y_{\rho}$. Set $E=\{\Sigma W: W$ is a bounded subset of $X\}$ and $D=\mathscr{A}(E)$, the subalgebra generated by $E$. Observe that if $W$ is a subset of $X$, it has a subset of size at most $c(B)$ with the same supremum. Hence, $|D| \leq \min \left(2^{<\operatorname{wd}(B)}, \sup \left\{\lambda^{c(B)}: \lambda<\operatorname{wd}(B)\right\}\right)$.

Theorem 3.1. There exists $a \in B^{+}$such that $D \mid a$ is dense in $B \mid a$. Consequently, $\mathrm{d}(B) \leq \min \left(2^{<\operatorname{wd}(B)}, \sup \left\{\lambda^{c(B)}: \lambda<\operatorname{wd}(B)\right\}\right)$.

Proof. By way of contradiction, we will assume $D$ is nowhere relatively dense. Based on this assumption, we prove two lemmas.

Lemma 3.2. For each $\rho<\kappa$ and $a \in B^{+}$, there exist nonzero $c<a$, an antichain $A$ in $B \mid(a-c)$, and a function $f: A \rightarrow \kappa$ such that

(i) $\forall p \in \operatorname{con}(X, c), \sup \left(f^{\prime \prime}(\operatorname{con}(A, p))\right)=\kappa$,

(ii) $\operatorname{con}\left(Y_{\rho}, c\right)=\operatorname{con}\left(Y_{\rho}, \Sigma A\right)$, and

(iii) $\Sigma A=a-c$.

Proof of the lemma. Fix $\rho<\kappa$ and $a \in B^{+}$. We will first show that $\exists c, A$, and $f$ such that conditions (i) and (ii) are satisfied. Since $D$ is nowhere relatively dense, by Observation $1.3, \exists$ nonzero $s<a$ such that $\operatorname{con}(D, s) \subset$ $\operatorname{con}(D, a-s)$. Let $U=\{x \in X: x \cdot s=0\}$ and $u=a \cdot \Sigma U$. Observe that $s \leq(-u) \cdot a$. Also note that if $x \in U, x \notin \operatorname{con}(X,(-u) \cdot a)$. Thus, we have $\operatorname{con}(X,(-u) \cdot a-s) \subset \operatorname{con}(X, s)$. By the homogeneous weak density of $X$, $\exists q \in X$ such that $0<q \cdot(-u) \cdot a \leq s$. Set $c=q \cdot(-u) \cdot a, T=\{x \in X: x \cdot c=0\}$ and $t=a \cdot \Sigma T$. For each $\alpha<\kappa$, set $T_{\alpha}=Y_{\alpha} \cap T$ and $t_{\alpha}=a \cdot \Sigma T_{\alpha}$ (if $T_{\alpha}=\varnothing$ set $\left.t_{\alpha}=0\right)$. Then $\forall \alpha<\kappa, t_{\alpha} \in D \mid a$. Also, observe that since $c \leq s$, we have $u \leq t$ and $(-t) \cdot a \leq-u \cdot a$. Set

$$
A=\left\{t_{\alpha}-\sum_{\tau<\alpha} t_{\tau}: \alpha>\rho \text { and } t_{\alpha}-\sum_{\tau<\alpha} t_{\tau} \neq 0\right\} .
$$

Define $f: A \rightarrow \kappa$ by $f\left(t_{\alpha}-\sum_{\tau<\alpha} t_{\tau}\right)=\alpha$. We show (i) by way of contradiction. Suppose $\exists p \in \operatorname{con}(X, c)$ such that $\sup \left(f^{\prime \prime}(\operatorname{con}(A, p))\right)<\kappa$. Then $\exists \alpha<\kappa$ such that $p \cdot t=p \cdot t_{\alpha}$. We have $p \cdot q \cdot a=p \cdot q \cdot t+p \cdot q \cdot a \cdot(-t) \leq p \cdot q \cdot t_{\alpha}+p \cdot q \cdot a \cdot(-u) \leq$ $p \cdot q \cdot t_{\alpha}+s$. Thus, setting $w=p \cdot q \cdot a \cdot\left(-t_{\alpha}\right)$, we have $w \in D \mid a$ and $0<w \leq s$. This contradicts the fact that $\operatorname{con}(D, s) \subset \operatorname{con}(D, a-s)$. To show (ii) we need to show $\operatorname{con}\left(Y_{\rho}, \Sigma A\right) \subset \operatorname{con}\left(Y_{\rho}, c\right)$. Suppose $x_{\pi} \in \operatorname{con}\left(Y_{\rho}, \Sigma A\right)$. Then $\exists \alpha>\rho \geq \pi$ such that $x_{\pi} \cdot\left(t_{\alpha}-\sum_{\tau<\alpha} t_{\tau}\right) \neq 0$ which implies $x_{\pi} \notin T_{\pi}$, i.e., $x_{\pi} \cdot c \neq 0$. Thus, $x_{\pi} \in \operatorname{con}\left(Y_{\rho}, c\right)$.

Let $\mathbf{P}$ consist of all triples $(c, A, f)$ such that (i) and (ii) are satisfied. (For convenience, $h \in \mathbf{P}$ may be expressed as $h=\left(\pi_{1}(h), \pi_{2}(h), \pi_{3}(h)\right)$. We have shown that $\mathbf{P}$ is nonempty. Partial order $\mathbf{P}$ by $(c, A, f) \leq^{*}\left(c_{1}, A_{1}, f_{1}\right)$ if and only if $c \leq c_{1}$, and $\forall t \in A, \exists t_{1} \in A_{1}$ such that $t \leq t_{1}$ and $f(t)=f_{1}\left(t_{1}\right)$.

If $Q$ is a chain in $\left(\mathbf{P}, \leq^{*}\right)$, set $c_{M}=\sum_{q \in Q} \pi_{1}(q) . \forall \alpha<\kappa$, set $h_{\alpha}=$ $\sum\left\{\Sigma f^{-1}(\alpha): f=\pi_{3}(q)\right.$ for some $\left.q \in Q\right\}$. (Take $h_{\alpha}=0$ if the preceding set is empty.) Setting $A_{M}=\left(h_{\alpha}\right)_{\alpha<\kappa}$, we have $A_{M}^{+}$is an antichain. Define the 
function $f_{M}: A_{M}^{+} \rightarrow \kappa$ by $f_{M}\left(h_{\alpha}\right)=\alpha$. Clearly, $\left(c_{M}, A_{M}^{+}, f_{M}\right)$ is a bound for $Q$.

Let $(c, A, f)$ be a maximal element of $\mathbf{P}$. We will show that (iii) is satisfied. If this were not so, then $0 \neq a_{1}=a-(c+\Sigma A)$. Choose nonzero $c_{1}<a_{1}$, an antichain $A_{1}$ in $B \mid\left(a_{1}-c_{1}\right)$, and a function $f_{1}: A_{1} \rightarrow \kappa$ such that (i) $\forall p \in$ $\operatorname{con}\left(X, c_{1}\right), \sup \left(f_{1}^{\prime \prime}\left(\operatorname{con}\left(A_{1}, p\right)\right)\right)=\kappa$ and (ii) $\operatorname{con}\left(Y_{\rho}, c_{1}\right)=\operatorname{con}\left(Y_{\rho}, \Sigma A_{1}\right)$. Then $(c, A, f)<^{*}\left(c+c_{1}, A \cup A_{1}, f \cup f_{1}\right)$. This contradicts maximality and completes the proof of the lemma.

Lemma 3.3. There exists an antichain $\left(c_{n}\right)_{n<\omega}$ such that $\left[\operatorname{con}\left(X, c_{n}\right)\right]_{n<\omega}$ is nondecreasing.

Proof of the lemma. For each $c \in B^{+}$we define the property $\sigma(c)$ as follows: $\sigma(c)$ : There exist an antichain $A$ in $B \mid(-c)$ and a function $f: A \rightarrow \kappa$ such that for each $x$ in $\operatorname{con}(X, c), \sup \left(f^{\prime \prime}(\operatorname{con}(A, x))=\kappa\right.$.

Applying Lemma 3.2 to the element 1, we know that there exists $c_{0} \in B^{+}$ such that $\sigma\left(c_{0}\right)$ holds. For each $n, 0<n<\omega$, we will choose $c_{n} \in B^{+}$such that the following conditions called $\varphi(n)$ hold:

(1) $\left(\sum_{k<n} c_{k}\right) \cdot c_{n}=0$,

(2) if $x \in X$ and $x \cdot\left(\sum_{k<n} c_{k}\right) \neq 0$, then $x \cdot c_{n} \neq 0$, and

(3) $\sigma\left(\sum_{k \leq n} c_{k}\right)$.

Fix $n, 0<n<\omega$. Assume $\left(c_{k}\right)_{k<n}$ have been constructed. By condition (3) of $\varphi(n-1), \sigma\left(\sum_{k<n} c_{k}\right)$ holds (if $n=1$ the preceding expression is merely $\left.\sigma\left(c_{0}\right)\right)$. There is an antichain $Z$ in $B \mid\left(-\sum_{k<n} c_{k}\right)$ and a function $v: Z \rightarrow \kappa$ such that if $x \in X$ and $x \cdot\left(\sum_{k<n} c_{k}\right) \neq 0$, then $\sup \left(v^{\prime \prime}(\operatorname{con}(Z, x))\right)=\kappa$. Using our Lemma 3.2, for each $z \in Z$ we choose nonzero $s_{z}<z$, an antichain $K_{z}$ in $B \mid\left(z-s_{z}\right)$, and a function $r_{z}: K_{z} \rightarrow \kappa$ such that

(i) $\forall p \in \operatorname{con}\left(X, s_{z}\right), \sup \left(r_{z}^{\prime \prime}\left(\operatorname{con}\left(K_{z}, p\right)\right)\right)=\kappa$,

(ii) $\operatorname{con}\left(Y_{v(z)}, s_{z}\right)=\operatorname{con}\left(Y_{v(z)}, \Sigma K_{z}\right)$, and

(iii) $\Sigma K_{z}=z-s_{z}$.

Observation. Let $\tau<\kappa$ and $z \in Z$. If $v(z) \geq \tau$ and $x_{\tau} \cdot z \neq 0$, then $x_{\tau} \cdot s_{z} \neq 0$. (This follows directly from (ii) and (iii).)

Set $c_{n}=\sum_{z \in Z} s_{z}$. Clearly, condition (1) of $\varphi(n)$ is satisfied. Consider $x_{\tau} \in X$ such that $x_{\tau} \cdot\left(\sum_{k<n} c_{k}\right) \neq 0$. By assumption, there is some element $z_{0} \in Z$ such that $x_{\tau} \cdot z_{0} \neq 0$ and $v\left(z_{0}\right) \geq \tau$. By the above observation, we have $x_{\tau} \cdot s_{z_{0}} \neq 0$ which implies $x_{\tau} \cdot c_{n} \neq 0$. Thus, condition (2) of $\varphi(n)$ holds. Set $A=\bigcup_{z \in Z} K_{z}$ and $f=\bigcup_{z \in Z} r_{z}$. Then $A$ is an antichain and $f$ is a function from $A$ into $\kappa$. Suppose $x_{\tau} \cdot\left(\sum_{k \leq n} c_{k}\right) \neq 0$. To demonstrate condition (3) of $\varphi(n)$ we first show that there exists $z_{1} \in Z$ such that $x_{\tau} \cdot s_{z_{1}} \neq 0$. Note that $\left(\sum_{k \leq n} c_{k}\right)=\sum_{z \in Z} s_{z}+\left(\sum_{k<n} c_{k}\right)$. If $x_{\tau} \cdot \sum_{z \in Z} s_{z} \neq 0$, then certainly there exists $z_{1} \in Z$ such that $x_{\tau} \cdot s_{z_{1}} \neq 0$. Otherwise, $x_{\tau} \cdot\left(\sum_{k<n} c_{k}\right) \neq 0$, and by assumption, 
there exists $z_{1} \in Z$ such that $x_{\tau} \cdot z_{1} \neq 0$ and $v\left(z_{1}\right) \geq \tau$, in which case, $x_{\tau} \cdot s_{z_{1}} \neq$ 0 . Observe that $r_{z_{1}}^{\prime \prime}\left(\operatorname{con}\left(K_{z_{1}}, x_{\tau}\right)\right)=f^{\prime \prime}\left(\operatorname{con}\left(K_{z_{1}}, x_{\tau}\right)\right) \subset f^{\prime \prime}\left(\operatorname{con}\left(A, x_{\tau}\right)\right)$. Since $x_{\tau} \in \operatorname{con}\left(X, s_{z_{1}}\right)$, we have $\sup \left(f^{\prime \prime}\left(\operatorname{con}\left(A, x_{\tau}\right)\right)\right) \geq \sup \left(r_{z_{1}}^{\prime \prime}\left(\operatorname{con}\left(K_{z_{1}}, x_{\tau}\right)\right)\right)=\kappa$. We have shown condition (3) of $\varphi(n)$ is satisfied. This completes the proof of the lemma.

To complete the proof of Theorem 3.1, let $\left(c_{n}\right)_{n<\omega}$ be as in Lemma 3.3. Then $\operatorname{con}\left(X, \sum_{n<\omega} c_{2 n}\right)=\operatorname{con}\left(X, \sum_{n<\omega} c_{2 n+1}\right)$. But, this contradicts the fact that $X$ is homogeneously weakly dense. Our assumption that $D$ is nowhere relatively dense is wrong. Finally,

$$
\mathrm{d}(B) \leq \min \left(2^{<\mathrm{wd}(B)}, \sup \left\{\lambda^{c(B)}: \lambda<\operatorname{wd}(B)\right\}\right)
$$

follows from homogeneity.

Remark. There are situations for which $2^{<\mathrm{wd}(B)}$ is less than

$$
\sup \left\{\lambda^{c(B)}: \lambda<\operatorname{wd}(B)\right\}
$$

and vice versa. For example, if $\operatorname{wd}(B)=c(B)=\omega_{1}$, then $\mathrm{CH}$ implies $2^{<\operatorname{wd}(B)}=$ $\omega_{1}$; however, $\sup \left\{\lambda^{c(B)}: \lambda<\operatorname{wd}(B)\right\} \geq \omega_{2}$. Also, if $B$ is c.c.c. and $\operatorname{wd}(B)=$ $\omega_{\omega}$, then $2^{\omega}<\omega_{\omega}$ implies $\sup \left\{\lambda^{c(B)}: \lambda<\operatorname{wd}(B)\right\}=\omega_{\omega}$ regardless of how big $2^{\omega_{n}}(0<n<\omega)$ are.

Corollary 3.4. $\mathrm{d}(B)=\mathrm{wd}(B)$ in $\mathrm{ZFC}+\mathrm{GCH}$.

Proof. For all infinite cardinals $\kappa, \kappa=2^{<\kappa}$ in $\mathrm{ZFC}+\mathrm{GCH}$.

\section{ACKNOWLEDGMENTS}

I thank my advisor, R. Daniel Mauldin, and Steve Jackson for their interest and suggestions concerning this work. In addition, I thank the referee for his suggestions in revising and improving this paper.

\section{REFERENCES}

1. K. Bozeman, Ph.D. thesis, University of North Texas, 1990.

2. M. Burke, Weakly dense subsets of the measure algebra, Proc. Amer. Math. Soc. 106 (1989), 867-874.

3. W. Just, unpublished manuscript.

4. S. Koppelberg, General theory of Boolean algebras: handbook of Boolean algebras, NorthHolland, Amsterdam, 1989.

Department of Mathematics, University of North Texas, P. O. Box 5116, Denton, TEXAS 76203 